\title{
The Mental Health Care Act: Stakeholder compliance with Section 40 of the Act
}

G Jonsson, FCPsych (SA)

M Y H Moosa, MMed (Psych), FCPsych (SA), MCFP

F Y Jeenah, MMed (Psych)

Department of Neurosciences, Division of Psychiatry, University of the Witwatersrand, Johannesburg

Objective. To determine compliance with Section 40 (1-3) of the Mental Health Care Act (MHCA) No 17 of 2002, viz. handing over custody by the South African Police Service (SAPS) of suspected mentally ill patients to medical services at Chris Hani Baragwanath Hospital (CHBH).

Methods. The study was a retrospective record review of patients who were 18 years and older, referred by SAPS to $\mathrm{CHBH}$. In accordance with Section 40 of the MHCA, a MHCA form 22 is completed when suspected mentally ill patients are handed over by SAPS to the medical services. MHCA forms 22 that were completed during the period July 2007 to December 2007 were obtained from hospital records and analysed to determine the compliance of SAPS and medical practitioners in completing them.

Results. During the study period, 708 of the 718 patients handed over by SAPS to the Emergency Department of $\mathrm{CHBH}$ were entered on MHCA forms 22. SAPS officials had correctly completed $86 \%$ of the forms, whereas the medical practitioners had only correctly completed $9.9 \%$ of the forms. Of the 718 patients handed over by SAPS, $319(44 \%)$ were discharged for outpatient care.

Conclusion. The findings of this study suggest that not all parties fully comply with Section 40 of the Mental Health Care Act No 17 of 2002. We suggest that the situation can be improved by training of all parties; amendments to the MHCA form 22; partnership between mental health care practitioners and members of SAPS; and combining resources towards implementing a crisis intervention model similar to that in other countries.

Worldwide, new laws have been enacted concerning the issues and profiles of mental health care users (MHCUs). These laws serve as an advocacy for MHCUs and play an extremely important part in protecting their health and human rights.
Included in these laws are the roles, responsibilities and terms of collaboration between the various stakeholders responsible for the care of MHCUs. ${ }^{1 \cdot 3}$

In South Africa, the new Mental Health Care Act (MHCA) ${ }^{4}$ was promulgated in 2002 and implemented in 2004. Similar to most legislation worldwide, ${ }^{1.3}$ this Act defines a collaborative and mutually co-operative relationship between the South African Police Services (SAPS), the mental health care practitioner (MHCP), the judiciary, and emergency medical services (EMS) at a local and national level. The Act serves to preclude the detention of acutely mentally ill users in police cells, and prioritises prompt access to local hospitals. It defines the role of the various stakeholders regarding transportation, hospitalisation and care of a MHCU within the criminal justice system. It also sets out a clear policy and working relationship between SAPS and medical services, especially concerning the humane handling of MHCUs when they are acutely ill. More specifically, Section 40 of the MHCA and its regulations ${ }^{5}$ state that if a member of the SAPS has reason to believe, either from personal observation or information obtained from a MHCP, that a person is mentally ill and is likely to inflict harm on himself or others, that member must apprehend the person and cause him to be either: (i) taken to an appropriate health establishment for assessment of his mental health status; or (ii) handed over into the custody of the head of health establishment (HHE) or any designated person to receive such a person. The handing over of custody includes the completion of a Mental Health Care Act (MHCA) form 22 by the HHE, SAPS member and the MHCP.

While the legislation is clear in its requirements, the compliance of various stakeholders in its implementation is still in question. In a study of outcomes of police responses to mental health emergencies, Steadman et al. ${ }^{6}$ concluded that current police training is inadequate to prepare police officers to identify and deal with the mentally ill. Police officers did not know how to recognise mental illness, how to deal with psychotic and violent behaviour, or what to do with someone trying to commit suicide. Teplin ${ }^{7}$ reported that police officers did not know what community resources were available, how to gain access to them, how to deal with their own emotional issues as a result of an extremely stressful career, or how to engage appropriately with mentally ill people. This lack of knowledge affects proper compliance with legislation. 
Teplin ${ }^{7}$ also reported that training goals about raising awareness of mental health issues were more successful than goals relating to influencing behavioural change. Furthermore, approaching police officers within their workplace allowed them to consider specific training needs and to create an environment where their attitudes and behaviours could be addressed. Similarly, persons with mental illness may also experience interactions with police officers in numerous ways. ${ }^{8}$ The manner in which police officers approach such situations will determine whether the mentally ill person co-operates or whether the situation becomes contentious or violent. Watson and Angell ${ }^{8}$ reported that police officers were more likely to arrest and incarcerate individuals with mental illness when: (i) there was evidence of a crime having been committed; (ii) the individual had a criminal history; (iii) they felt that the individual would be inadmissible to a hospital; (iv) public encounters exceeded the community's tolerance for defiant behaviour; and ( $v$ ) it was likely that the person would continue to be a problem.

It is evident that educational seminars for improving police officers' knowledge of mental illness and training in their roles concerning mental health care legislation should be provided. 9,10

As the MHCA of South Africa is still in its infancy, there are very few published data on this issue; hence the opportunity and need for this study, the purpose of which is to determine the level of compliance of SAPS and MHCPs in the apprehension, disposition and care of suspected mentally ill patients.

\section{Methods}

At Chris Hani Baragwanath Hospital $(\mathrm{CHBH})$, members of SAPS transfer all suspected mentally ill patients to the medical officer (MO) at the Emergency Department (ED). The MHCA form 22 serves to formalise this process, being used to record information at all steps in the procedure regarding the apprehension, handover and physical condition of the MHCU.

Following hand-over, the MO obtains a history, examines the patient and decides on further management. The physical examination serves to exclude medical causes of the patient's psychiatric symptomatology. After completing the assessment, the $\mathrm{MO}$ in the emergency room completes the form 22 (including the sections relating to physical condition of the MHCU at handover and the physical examination findings) and may then refer the MHCU to the medical admission ward (MAW). The HHE at $\mathrm{CHBH}$ designates the ED's admitting $\mathrm{MO}$ to complete this section of form 22.
In the MAW, all MHCUs are fully assessed by the ward's psychiatric registrar, and a decision is taken to either admit the user to a psychiatric ward or discharge the patient home from the MAW. Copies of all psychiatric notes are placed in the patient's file, together with a copy of the form 22, which is stored in the Psychiatry Department's filing room.

Our study was a retrospective record review of patients referred by SAPS to the ED at CHBH while using the form 22. All MHCUs referred by SAPS were included if: (i) a form 22 was in the patient's psychiatric file; (ii) the patient was $\geq 18$ years old; and (iii) the patient had been referred during the study period (July December 2007). All forms 22 completed during the period were obtained from patients' psychiatric files kept in the Department of Psychiatry's filing room (Fig. 1).

The following information was obtained from the forms 22 and entered onto a data capturing sheet by the investigator: (i) the SAPS member's rank, initial and surname; (ii) date and time; (iii) reasons for apprehending the MHCU; (iv) the MHCU's next of kin's contact details; and ( $V$ ) whether the MHCP recorded details of the MHCU's physical condition at the time of hand-over. The MHCU's forensic history was also obtained from the psychiatric file.

Each data sheet was assigned a unique identifying case number for filing purposes. Some participants had more than one visit during the study period; each such visit was considered as a separate case.

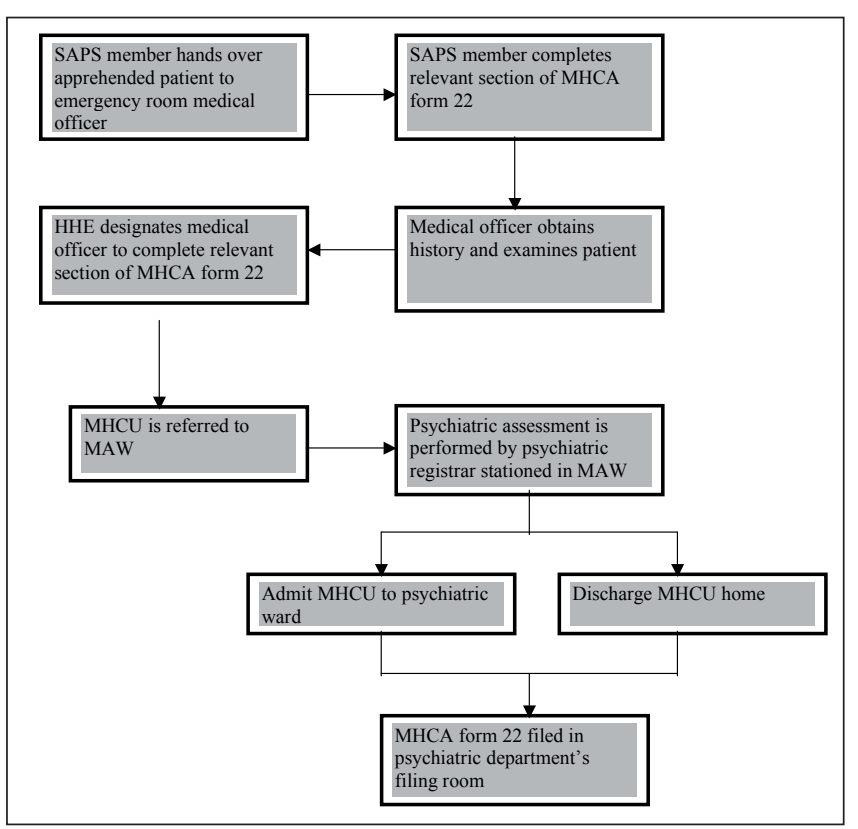

Fig. 1. Referral process and completion of MHCA form 22. 
Descriptive statistics were computed as means and frequencies (count and percentages). The study was submitted to the University of the Witwatersrand's Human Research Ethics Committee (HREC) and approval was obtained. All MHCUs' details remained anonymous, and confidential information was not recorded on the data sheet.

\section{Results}

During the 6-month study period, 2754 patients were admitted to the MAW. Members of SAPS referred $718 \mathrm{MHCUs}$ to the $\mathrm{CHBH}$ ED. Forms and psychiatric hospital notes of only 708 referrals could be traced and included in the study. Of the $708 \mathrm{MHCA}$ forms completed, $87.4 \%(N=619)$ included SAPS officials' rank, initials and surname. Date and time were recorded on $98.6 \%$ $(\mathrm{N}=698$ ) of the forms. Reasons for suspecting mental illness and apprehending $\mathrm{MHCUs}$ were recorded on 86.2\% (N=610) of the forms. Details of MHCUs' next of kin were recorded on 96.2\% ( $\mathrm{N}=681$ ) of the forms (Table I). Details regarding MHCUs' physical condition on arrival at the ED were recorded by the MHCP on only $9.9 \%(\mathrm{~N}=70)$ of the forms. Overall, only $5.4 \%$ $(\mathrm{N}=38$ ) of the forms 22 were fully completed by all parties (SAPS, MHCP, HHE (or designated person)) concerned (Table I). MHCUs' forensic history was recorded as positive in only $6.6 \%$ of cases, and was unknown in $85.9 \%$ (Table II).

\section{Discussion}

\section{Rate of police referrals}

This study found that approximately 1 in $4(26 \%)$ referrals to $\mathrm{CHBH}$ were by the SAPS, which is a higher rate than most other published studies. Kneebone et al. ${ }^{11}$ reported that, over a 21 -month period in South Australia, police referrals constituted $9.1 \%$ of all referrals from the community, and $9.9 \%$ of total

\begin{tabular}{lll} 
Table II. Distribution of forensic history \\
\hline \\
$\begin{array}{lll}\text { Number } \\
\text { Forensic history }\end{array}(\mathrm{N}=708)$ & $\%$ \\
\hline Positive & 47 & 6.64 \\
Negative & 53 & 7.49 \\
Unknown & 608 & 85.88 \\
\hline
\end{tabular}

hospital admissions. Similarly, Bruffaerts et al., ${ }^{12}$ in their study on the epidemiological profile of patients consulting the psychiatric emergency team of a Belgian university hospital, found that $8.8 \%$ of all patients were police referrals. However, Knott et al., ${ }^{13}$ in their study of 3701 patients presenting to Victoria EDs in Australia, reported that $17.6 \%$ were police referrals, which was significantly higher than the figure for South Australia.

Possible reasons for the high figures in this study population could be sociopolitical. $\mathrm{CHBH}$ is the only referral institution in Soweto for acute psychiatric patients, and serves a vast urban population. This study population has a high unemployment rate (estimated at $23 \%$ ) and is largely socially disadvantaged - a legacy from the previous regime in South Africa. ${ }^{14,15}$ Families of mentally ill patients often lack the means to transport them to the only facility in the area. Furthermore, an inadequate EMS /only 0.4 ambulances per 100000 population) limits the availability of ambulances to transport such patients to the hospital. ${ }^{16}$ This is further compounded by reluctance on the part of emergency medical services (EMS) personnel to transport these patients by ambulance, as they are regarded as violent and aggressive. As a result, the SAPS are inappropriately utilised by the families of mentally ill individuals to transport them to the hospital, hence the higher police referral rate. In America, however, it is reported that it is the police who are often reluctant to intervene where mental

\begin{tabular}{|c|c|c|}
\hline Sections of MHCA form 22 & Number of forms completed ( $N=708)$ & $\%$ of forms completed \\
\hline \multicolumn{3}{|l|}{ SAPS } \\
\hline Rank, initial and surname & 619 & 87.43 \\
\hline Date and time & 698 & 98.59 \\
\hline Reasons for apprehending the $\mathrm{MHCU}$ & 610 & 86.16 \\
\hline MHCU's next of kin - all details & 681 & 96.19 \\
\hline Name only & 638 & 90.11 \\
\hline Contact details only & 658 & 92.94 \\
\hline \multicolumn{3}{|l|}{ MHCP } \\
\hline MHCU's physical condition & 70 & 9.89 \\
\hline All sections completed & 38 & 5.37 \\
\hline
\end{tabular}


illness is concerned; they frequently under-identify mental illness, and prefer not to transport individuals to hospital because of the length of time spent in waiting for admission. ${ }^{17,18}$

A large proportion of the mentally ill first come to the attention of the police; it is the latter's decision, then, to refer the patient to either the criminal justice or mental health system. Lamb and Weinberger ${ }^{19}$ reported that the police tend to refer to the criminal justice system because the offender will be dealt with in a more systematic way (will be seen by a mental health professional, receive psychiatric evaluation and treatment) in prison. In South Africa, prisons and police cells are not frequented by psychiatrists who routinely evaluate offenders, and it is therefore easier for SAPS personnel to deposit the offender at mental health services and return to their duties; this situation may also be due to the fact that no other appropriate community alternatives are available.

\section{Stakeholder compliance with the MHCA}

It is evident from this study that the SAPS are more compliant about completing form 22 and the MHCA regulations compared with MHCPs in the ED at $\mathrm{CHBH}$. Although training in the specifics of the MHCA are not formally carried out for SAPS, they may be more compliant in completing form 22 because it forms part of their standard operating procedures (SOPs), regardless of their understanding of the MHCA. A similar SOP does not exist for medical practitioners at this hospital.

Contrary to our findings, Lynch et al. ${ }^{20}$ in their study assessing the level of knowledge of Section 136 of the Mental Health Care Act 1983 of the UK, found that accident and emergency officers had the highest level of working knowledge of the Act. Nearly 1 1\% of the police officers in their study failed even to recognise that a person has to appear to be suffering from a mental disorder to fall under this Act. They also reported that only $10.3 \%$ of accident and emergency staff, and $22.8 \%$ of police, had received any formal training in carrying out the procedures of the Act.

Our study also revealed that the physical condition of MHCUs at the time of hand-over by SAPS was recorded in only 10\% of all referrals. It is possible that the MHCPs were either not examining patients properly or were not entering their findings on form 22, which may be due to high patient loads at the emergency department of $\mathrm{CHBH}$. The department attends to approximately 500 emergency room visits in 24 hours and is severely under-resourced (average of 4 medical officers on duty). Violent or aggressive patients are quickly restrained and routed to psychiatric services without a complete physical examination. Patient violence and hostility and a lack of appropriate skills and equipment may also explain why MOs do not examine all referred patients. Furthermore, the stigma surrounding mental illness, and perhaps $\mathrm{MO}$ perceptions that physical examinations are not important in the mentally ill, could also be contributing to this phenomenon. As a result of failure on the part of admitting MOs to perform a proper physical examination, the burden of excluding medical illness is transferred to the psychiatric registrar. It is often very difficult to re-route these patients to appropriate medical care once they have been labelled as mentally ill. More importantly, documented evidence of the physical state of the user on hand-over before admission is crucial when claims of physical abuse while in the care of SAPS or the hospital are raised by the $\mathrm{MHCU}$ or other parties concerned.

We also observed that only $6.6 \%$ of patients referred by SAPS in this study reported a previous forensic history. This is contrary to other reported studies. Kneebone et al." reported that $40 \%$ of police referrals had a forensic history, in which assault and theft were the most common offences. Kisely et al. ${ }^{21}$ reported a prevalence of convictions of lifetime offences of between 21\% and $23 \%$. The majority of MHCUs in this study had no forensic history recorded. Although a forensic history does not affect the decision to admit a patient las admission depends on the patient's presenting mental state), doctors at $\mathrm{CHBH}$ are admitting patients referred by SAPS, yet fail to obtain information on their forensic history. Mental health services at $\mathrm{CHBH}$ and other general hospitals are usually not fully equipped to deal with potentially aggressive or violent patients, which could pose problems with regard to the safety of staff and other patients. It may also be helpful to determine where the patient is discharged following admission; this finding highlights the necessity for hospital managers to insist on the need to upgrade skills and infrastructure at general hospitals, to deal more effectively with potentially violent patients.

It was also noted that family members usually did not accompany MHCUs to the ED; therefore, no collateral information (such as reason for the episode, or relapse of psychiatric symptoms) is available to the emergency doctor. We recommend that the accompaniment of family members should be insisted upon by the SAPS and should be written into SAPS SOPs.

\section{Training recommendations}

Training in psychiatry and the MHCA has been included in undergraduate medical training over the past few years. However, it has only recently been incorporated into the newly established postgraduate degree in emergency medicine. In South Africa, because of a lack of emergency medicine specialists, general practitioners often form the bulk of emergency room staff. Consequently, most current emergency doctors lack the 
knowledge, understanding, skills and competence to implement the MHCA and to examine and assess acutely psychotic patients. Academics need to place a special emphasis on training in carrying out the procedures of the MHCA and the management of violent patients for undergraduate and postgraduate medical students. At the same time, it is also necessary to provide regular updates for current emergency room staff, perhaps as part of their in-service training. In addition, regular audits of the processes and procedures need to be carried out in all hospital EDs to assist in establishing protocols specific to the hospital situation.

Vermette et al. ${ }^{22}$ surveyed Massachusetts police officers' training for their work with mentally ill persons. They found that $90 \%$ of respondents reported that the topic of mental illness was '... either fairly or very important to their work' and that $70 \%$ had received post-academy mental health training. It has also been reported that training for police officers should include: (i) general classification of mental disorders; (ii) management of aggressive or violent patients; (iii) becoming familiar with local criteria used for involuntary hospitalisation; and (iv) information regarding access to resources other than hospitalisation, e.g. substance abuse and rehabilitation centres. ${ }^{22,23}$ This is important in the South African context as the inclusion of mental illness does not form part of SAPS training. Regardless of the method, training is imperative for SAPS members.

Mental health and law enforcement professionals need to synergise to provide each other with the most effective means of managing mentally ill persons. ${ }^{20}$ Munetz et al. ${ }^{23}$ studied various models in developing their programme, and then used centres of excellence to distribute it throughout their respective states. Once communities adopted their team programme, a 'train the trainer' model was initiated. Communities were invited to send a team representing law enforcement, mental health and advocacy groups to the training course. In 1988, in Memphis, USA, a crisis intervention team model was started. The team comprised police officers who received special mental health training to provide crisis intervention services and act as liaisons with the mental health system. The Memphis model is an example of policebased police response teams. Other models include police-based mental health response teams that involve mental health clinicians working as civilian employees of the police department, and mental health-based mental health response teams that involve partnerships with mobile mental health teams that are part of community mental health centres. ${ }^{6}$

EDs are used as a drop-off centre by police in crisis situations when no formal crisis intervention team or model exists. This inefficient approach is used at $\mathrm{CHBH}$ and most other general hospitals.
We recommend that a combination or adaptation of the above recognised and effective models needs to be developed for South Africa; this will require involvement by all participants in the programme, plus a substantial injection of financial and human resources for its implementation. Further research is necessary to develop such an appropriate model.

Our retrospective study design may be viewed as a limitation in this study. Some data might not have been recorded in case notes. However, every reasonable effort was made to collate information from the forms 22 and hospital notes. The majority of our patients' records contained all the data required, and the conclusions drawn are therefore reliable. This study may not be generalisable to other provinces/areas with dissimilar procedures of SAPS referrals, emergency evaluations at the ED, or to hospitals with different referral bases or populations.

\section{Conclusion}

The findings of this study suggest that not all stakeholders are fully compliant with the procedure as set out in the new Mental Health Care Act No 17 of 2002. Differences in knowledge may be a result of differences in understanding of various roles played in different stages of the Act. We recommend that implementation of Section 40 of the Act may be improved by: (i) providing training to all stakeholders; (ii) making amendments to the MHCA form 22 (e.g. use of checkboxes may increase the likelihood of all components being completed appropriately without delaying the police officers); (iii) increasing the quality of the partnerships of all stakeholders concerned; and (iv) trying to combine the resources of the two departments towards implementing a crisis intervention model similar to that employed in other countries.

\section{References}

1. Section 136/135 Mental Health Care Act 1983 - Policy and Procedure Guidelines. Pretoria: Department of Health. http://www.devon.gov.uk/deve/ section_v_m_health_act_1983_sections_136_135.pdf laccessed 29 February 2008).

2. Mental Health: Mental Health Act Amendments. Ontario, Canada: Ministry of Health and Long-erm Care. hitp//www.health.gov.on.ca/English/public/pub/ mental/faq.html. (accessed 23 June 2008).

3. Mental Health Act 1993 No 59 of 1993. Pretoria: Department of Health. hittp:// www.health.wa.gov.au/mhareview/resources/legislation/SA_Mental Health Act_1 1993.pdf (accessed 22 June 2008).

4. Mental Health Care Act No 17 of 2002. Pretoria: Department of Health, 2004. http://www. info.gov.za/gazette/acts/2002 (accessed 29 February 2008).

5. Mental Health Care Act No. 17 of 2002: Regulations; Chapter IX, Section 66: 7 (k). Pretoria: Department of Health, 2004. http://www.info.gov.za/gazette/ acts/2002 (accessed 29 February 2008).

6. Steadman HJ, Deane MW, Borum R, Morrissey J. Comparing outcomes of major models of police responses to mental health emergencies. Psychiatr Serv 2000; 51 645-649

7. Teplin LA. The criminalization of the mentally ill: speculation in search of data Psychol Bull 1983; 94: 54-67

8. Watson AC, Angell B. Applying procedural justice theory to law enforcements response to persons with mental illness. Psychiatr Serv 2007; 58: 787-793. 
9. Goldschalx SM. Effect of a mental health education programme upon police officers. Res Nurs Health 1983; 7: 111 .

10. Pinfold V, Huxley P, Thornicroft G, Farmer P, Toulmin H, Graham T. Reducing psychiatric stigma and discrimination: Evaluating an educational intervention with the police force in England. Soc Psychiatry Psychiatr Epidemiol 2003; 38: 337-344.

1 1. Kneebone P, Rogers J, Hafner RJ. Characteristics of police referrals to a psychiatric emergency unit in Australia. Psychiatr Serv 1995; 46: 620-622.

12. Bruffaerts R, Sabbe M, Demyttenaere K. Attenders of a university hospital psychiatric emergency service in Belgium. General characteristics and gender differences. Soc Psychiatry Psychiatr Epidemiol 2004; 39: 146-153.

13. Knott JC, Pleban A, Taylor D, Castle D. Management of mental health patients attending Victorian emergency departments. Aust NZJ Psychiatry 2007; 41: 759767.

14. PO210 - Labour Force Survey (LFS). Pretoria: Statistics South Africa http://www statssa.gov.za/keyindicators/keyindicators.asp (accessed 16 August 2008).

15. Provincial Profile 1999: Gauteng. Statistics South Africa. Health Systems Situation Analysis Report. Pretoria: Statistics South Africa, 2003. http://www.doh.gov.za/ facts/eusites/sekhukhune04.pdf laccessed 16 August 2008
16. Lund C, Flischer AJ. Norms for mental health services in South Africa. Soc Psychiatry Psychiatr Epidemiol 2006; 41 : 587-594.

17. Clark M. Emergency medicine in the new South Africa. Ann Emerg Med 1999; 32 367-372

18. Green TM. Police as frontline mental health workers. The decision to arrest or refer to mental health agencies. Int J Law Psychiatry 1997; 20: 469-486.

19. Lamb HR, Weinberger LE. Persons with severe mental illness in jails and prisons: A review. New Dir Ment Health Serv 2001; 90: 29-49

20. Lynch RM Simpson M, Higson M Grout P. Section 136, The Mental Health Act 1983; levels of knowledge among accident and emergency doctors, senior nurses, and police constables. J Emerg Med 2002; 19: 295-300.

21. Kisely SR, Xiao J, Preston NJ. Impact of compulsory community treatment on admission rates. Br J Psychiatry 2004; 184: 432-438.

22. Vermette HS, Pinals DA, Appelbaum PS. Mental health training for law enforcement professionals. J Am Acad Psychiatry Law 2005; 33: $42-46$.

23. Munetz MR, Morrison A, Krake J, Young B, Woody M. Statewide implementation of the crisis intervention team programme: The Ohio model. Psychiatr Serv 2006; 57: 1569-1571. 